\title{
Characteristics and upregulation of antioxidant capacity of fermented pueraria starch production wastewater with kombucha consortium
}

\author{
Zhixin Lu, Hongting Jiang, Zhongqiang Chen, Yin Zhai and Fahu Yuan* \\ School of medicine, Jianghan University, Wuhan, Hubei, China
}

\begin{abstract}
Pueraria lobata is a traditional plant used for both medicine and food. Pueraria starch has a long history of folk consumption in China. The processing of pueraria starch produces a lot of waste water and is often discarded, but it contains rich nutrients and is suitable for the growth of microorganisms. In this study, pueraria starch processing wastewater was used as a new fermentation substrate for kombucha fungus to develop a new functional beverage. After 8 days of static fermentation in $28{ }^{\circ} \mathrm{C}$, the $\mathrm{pH}$ of kombucha fermented with pueraria starch processing wastewater as substrate decreased to 2.78 , the total acid concentration was $0.158 \mathrm{~mol} / \mathrm{L}$, and the mass concentration of reducing sugar decreased to $2.05 \mathrm{mg} / \mathrm{mL}$. The pueraria starch production wastewater before and after fermentation was extracted with $80 \%$ methanol and the mass concentration of total flavonoids was determined. The antioxidant activity of pueraria starch processing wastewater before and after fermentation was analyzed by using three antioxidant models: DPPH free radical scavenging ability, ABTS cationic free radical scavenging ability and ferrous ion reducing power. The results showed that after 7 days of fermentation, the mass concentration of total flavonoids in kombucha produced by the pueraria starch processing wastewater was $268.45 \mathrm{mg} / \mathrm{L}$. The antioxidant activity of fermented kombucha beverage was significantly higher than that of unfermented pueraria starch processing wastewater. This study provides a new way for the resource utilization of Pueraria starch processing wastewater.
\end{abstract}

\section{Introduction}

Kombucha is a pure natural health drink with sweet and sour taste, which has certain health effects on a variety of diseases. It has been used in Asia for more than 2000 years. The traditional preparation method of kombucha fungus is natural fermentation with tea sugar water as the substrate, adding the kombucha fungus liquid and bacterial membrane. The functional characteristics of kombucha fungi are mainly determined by the active microorganisms and metabolites of tea extracts contained in fermentation broth. Due to the differences in bacterial species and culture methods, the metabolic pathway of kombucha bacteria is complex, and the types and contents of functional components in the obtained bacterial liquid are also different. Its functional characteristics are mainly attributed to the organic acids, phenols and other antioxidant substances produced in the fermentation process[1,2].

Pueraria lobata is a plant approved for both medicinal and edible use in China. The main active components of puerariae were isoflavones, dozens of isoflavones have been isolated and identified from pueraria root, mainly including puerarin, daidzein, daidzein, genistein, formononetin and so on. Pueraria isoflavones have the function of phytoestrogens to dilate coronary arteries and vessels, as well as the activities of anti-tumor, lowering blood glucose, lowering blood lipid and anti-oxidation. Pueraria isoflavone is a unique isoflavone in Pueraria, which has been widely used in the clinical treatment of cardiovascular and cerebrovascular system diseases, diabetes, fundus diseases and tumors[3].

Pueraria starch has a long history of being eaten by Chinese people. In recent years, large-scale processing enterprises began to appear in the production of pueraria starch, but traditional processing technology was generally adopted. In the process of starch production, large amount of water consumption led to environmental pollution, and active ingredients such as isoflavones from pueraria were lost with water discharge. Microbial fermentation may provide a new way for the utilization of pueraria starch processing wastewater. The purpose of this study is to develop kombucha fermentation beverage with pueraria starch production wastewater(PSPW) as the substrate, so as to realize the utilization of resources, reduce pollutant discharge and bring good economic benefits to the development enterprises of pueraria.

\footnotetext{
* Corresponding author: yuanfh@jhun.edu.cn
} 


\section{MATERIALS AND METHODS}

\subsection{Preparation of kombucha fermentation seeds}

Black tea was added to boiling water at $5 \mathrm{~g} / \mathrm{L}$, boiled for $5 \mathrm{~min}$, and white sugar with mass fraction of $10 \%$ was added, which was fully dissolved and filtered. After the tea sugar water was cooled to room temperature, kombucha fungus was added with mass fraction of $10 \%$, and incubated at $28{ }^{\circ} \mathrm{C}$ for $8 \mathrm{~d}$. The fermented kombucha bacteria were used for subsequent fermentation of PSPW.

\subsection{Preparation of PSPW fermented by kombucha fungus}

PSPW was sterilized at $108{ }^{\circ} \mathrm{C}$ for $15 \mathrm{~min}$, then cooled to room temperature. In a sterile environment, kombucha fungi were added at $10 \%$ mass fraction, and incubated at $28{ }^{\circ} \mathrm{C}$ for $7 \mathrm{~d}$. Samples were taken everyday in aseptic operation.

\subsection{Determination of $\mathrm{pH}$ and total acid concentration}

The change of $\mathrm{pH}$ during fermentation was measured by $\mathrm{pH}$ meter. The PSPW was titrated with $0.1 \mathrm{~mol} / \mathrm{L} \mathrm{NaOH}$ solution, and the total acid concentration was calculated as shown in Equation (1).

$$
C_{2}=\frac{C_{1} \times\left(V_{1}-V_{2}\right)}{V_{3}}
$$

In the Equation (1), $C_{2}$ is the total acid concentration (mol/L) of fermented PSPW; $C_{1}$ is the concentration of $\mathrm{NaOH}$ solution $(\mathrm{mol} / \mathrm{L}) ; V_{1}$ is the volume $(\mathrm{mL})$ of the fermentation broth titrated by $\mathrm{NaOH} . V_{2}$ is the volume $(\mathrm{mL})$ of the fermentation broth before titration with $\mathrm{NaOH} . V_{3}$ is the volume $(\mathrm{mL})$ of fermentation broth to be measured.

\subsection{Determination of reducing sugar concentration}

Refer to the method of Sengupta et al.[4], 3,5dinitrosalicylic acid colorimetric method was used to determine the mass concentration of reducing sugar in yellow pulp fermentation process.

\subsection{Determination of mass concentration of total phenols and total flavonoids}

Samples were extracted with methanol solution with a volume fraction of $80 \%$. Folin-phenol colorimetry was used to measure the samples according to the method of Chakravorty et al. [5]. The total phenol mass concentration was determined by gallic acid mass, calculated according to the standard curve. The mass concentration of total flavonoids was determined by referring to the method of Jia Zhishen et al.[12], which was calculated by rutin mass according to the standard curve.

\subsection{Antioxidant capacity in vitro}

\subsubsection{DPPH free radical scavenging ability was determined}

With reference to the method of Ding Yangru et al. [13], samples with different mass concentrations and tocopherol were prepared, and $2 \mathrm{~mL}$ DPPH solution $(0.5$ $\mathrm{mmol} / \mathrm{L}$ ) was added to $2 \mathrm{~mL}$ samples, shaken well, and reacted for $30 \mathrm{~min}$ in dark at room temperature. The absorbance DPPH free radical scavenging rate $\left(\mathrm{SR}_{\mathrm{DPPH}}\right)$ was measured at $517 \mathrm{~nm}$ wavelength and calculated as shown in Equation (2).

$$
\operatorname{SRDPPH}_{(\%)}\left(1-\frac{A_{\text {sample }}-A_{\text {blank }}}{A_{D D P H}}\right) \times 100
$$

In the Equation (2), $A_{D P P H}$ is the absorbance of DPPH and methanol solution; $A_{\text {sample }}$ is the absorbance of DPPH and sample solution; $A_{\text {blank }}$ is the absorbance of the sample and the methanol solution

\subsubsection{ABTS cationic radical scavenging ability}

ABTS solution of $7 \mathrm{mmol} / \mathrm{L}$ and potassium persulfate solution of $2.45 \mathrm{mmol} / \mathrm{L}$ were prepared and mixed by volume ratio of $1: 2$ and placed in dark environment for $16 \mathrm{~h}$. Before use, ethanol was used to dilute ABTS solution to make its absorbance at $734 \mathrm{~nm}$ range from 0.70 to 0.02 . Sample of different mass concentrations and $0.3 \mathrm{~mL}$ of tocopherol solution were prepared, and $1.2 \mathrm{~mL}$ of ABTS solution was added to react at room temperature for $6 \mathrm{~min}$ to determine the absorbance at $734 \mathrm{~nm}$ wavelength. The ABTS cationic radical scavenging rate was calculated as shown in Equation (3).

$$
\operatorname{SR} \text { ABts }(\%)=\left(1-\frac{A_{\text {sample }}}{A_{\text {control }}}\right) \times 100
$$

In the Equation (3), $A_{\text {sample }}$ is the absorbance of ABTS and sample solution; $A_{\text {control }}$ is the absorbance of ABTS and methanol solution.

\subsubsection{Determination of reducing power of ferrous ions}

A $0.3 \mathrm{~mol} / \mathrm{L}$ acetic acid buffer ( $\mathrm{pH}$ 3.6) was prepared, and $10 \mathrm{mmol} / \mathrm{L}$ TPTZ solution and $20 \mathrm{mmol} / \mathrm{L} \mathrm{FeCl}_{3}$ solution were prepared with $40 \mathrm{mmol} / \mathrm{L} \mathrm{HCl}$ solution. The above solutions were mixed at a volume ratio of 10:1:1 to prepare ferrous ion reduction solution. Samples of different concentrations and $0.2 \mathrm{~mL}$ of tocopherol solution were prepared, and $1 \mathrm{~mL}$ of ferrous ion reduction solution was added, placed at $37^{\circ} \mathrm{C}$ for $20 \mathrm{~min}$, and the absorbance at $593 \mathrm{~nm}$ wavelength was measured. The standard solution of gradient $\mathrm{FeSO}_{4}$ (100 1400 $\mu \mathrm{mol} / \mathrm{L})$ was prepared. The absorbance was measured at $593 \mathrm{~nm}$ and the standard curve was plotted, the reduction power of ferrous ions is expressed by calculating the concentration of ferrous ions through the standard curve. 


\subsection{Data statistics and analysis}

The experimental data were the mean of 3 replicates, and the results were expressed as mean \pm standard deviation. SPSS software was used for statistical analysis, Duncan's method was used for multiple comparisons, and GraphPad Prism software was used for plotting.

\section{Results}

\subsection{Physicochemical and microbiological characteristics of kombucha beverages}

Changes of $\mathrm{pH}$ value and total acidity in the fermentation process of kombucha are shown in Figure 1 and Figure 2. After PSPW fermentation, the $\mathrm{pH}$ value decreased rapidly in the first 3 days, then decreased slightly and stabilized after 5 days of fermentation (Fig. 1). On the contrary, titratable acidity content continued to increase until the 6th day, with the most obvious increase from $0.066 \mathrm{~mol} / \mathrm{L}$ to $0.172 \mathrm{~mol} / \mathrm{L}$ between the $3 \mathrm{rd}$ and 6 th days, and slightly decreased to $0.158 \mathrm{~mol} / \mathrm{L}$ on the 8th day (Fig. 2).

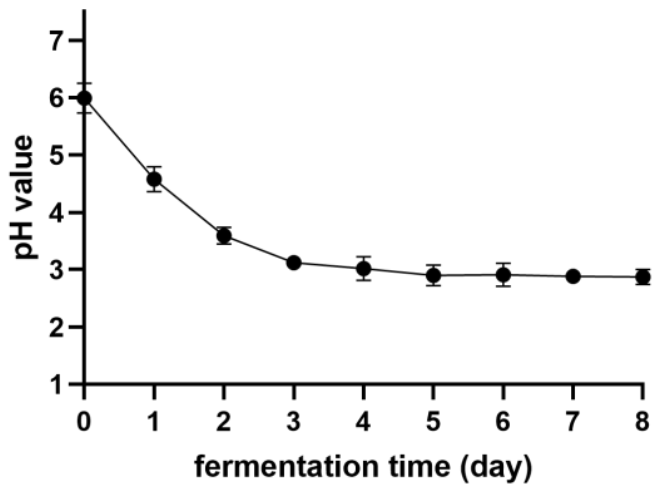

Fig. 1 Changes in $\mathrm{pH}$ during kombucha fermentation. Data were shown as the mean $\pm \operatorname{SD}(n=3)$.

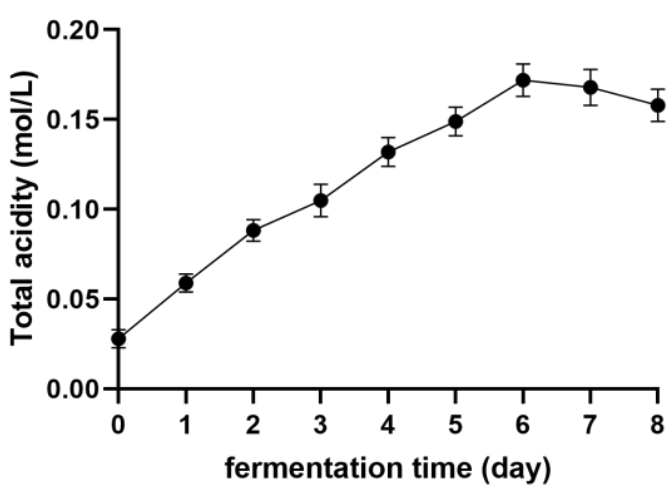

Fig. 2 Changes in total acidity during kombucha fermentation. Data were shown as the mean $\pm \operatorname{SD}(n=3)$.

As shown in Figure 3, the reducing sugar concentration increased slightly on the first day, decreased sharply from $10.55 \mathrm{mg} / \mathrm{mL}$ to $1.86 \mathrm{mg} / \mathrm{mL}$ on the first to the third day, and then fluctuated between 1.73 and $2.05 \mathrm{mg} / \mathrm{mL}$.

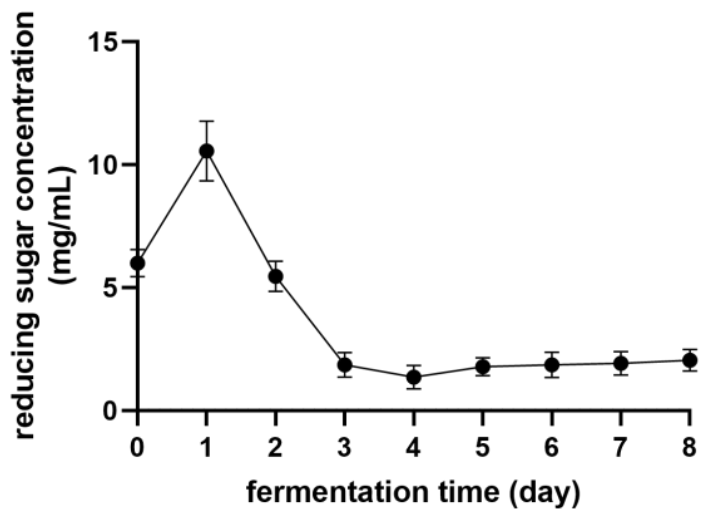

Fig. 3 Changes in reducing sugar concentration during kombucha fermentation. Data were shown as the mean \pm SD (n $=3$ ).

\subsection{Changes in the concentrations of total phenols and total flavonoids}

As shown in figure 4 , the concentrations of total phenols and total flavonoids in kombucha beverages were significantly increased. After fermentation, the concentration of total phenol in PSPW increased from $256.22 \mathrm{mg} / \mathrm{L}$ to $653.15 \mathrm{mg} / \mathrm{L}$ on the 8th day, and the mass concentration of total flavonoids continued to increase from $152.38 \mathrm{mg} / \mathrm{L}$ to $359.65 \mathrm{mg} / \mathrm{L}$ on the 8 th day. The results showed that phenols and flavonoids were newly formed in PSPW after fermentation.

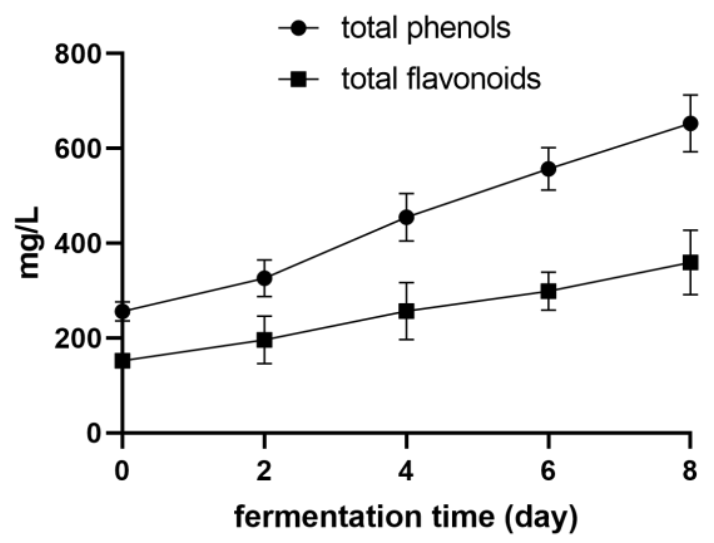

Fig. 4 Changes in the concentrations of total phenols and total flavonoids during kombucha fermentation. Data were shown as the mean $\pm \mathrm{SD}(\mathrm{n}=3)$.

\subsection{Antioxidant capacity in vitro}

As shown in Figure 5A, compared with unfermented PSPW, the DPPH free radical scavenging ability of fermented PSPW was significantly improved. When the sample concentration was $0.25-10 \mathrm{mg} / \mathrm{mL}$, the DPPH free radical scavenging rate of unfermented PSPW increased from $10.05 \%$ to $60.50 \%$, while that of kombucha increased from $25.38 \%$ to $95.45 \%$. The 
results indicated that the fermentation process played an important role in improving the DPPH free radical scavenging ability.

As shown in Fig. 5B, when the concentration of PSPW was between 0.25 and $6 \mathrm{mg} / \mathrm{mL}$, the cationic radical scavenging ability of ABTS was significantly improved before and after fermentation. When the concentration was more than $6 \mathrm{mg} / \mathrm{mL}$, the scavenging ability of ABTS cationic radicals was maintained at about $60 \%$ and $90 \%$, respectively. This means that when the concentration exceeds the upper limit of saturation, the scavenging ability of phenolic compounds against ABTS cationic radicals no longer increases.

As shown in Fig. 5C, the ferrite reducing power of unfermented PSPW and kombucha is proportional to the sample concentration. The ferric concentration of kombucha increased from $29.95 \mu \mathrm{mol} / \mathrm{L}$ to 500.03 $\mu \mathrm{mol} / \mathrm{L}$, while the unfermented PSPW only increased from $10.25 \mu \mathrm{mol} / \mathrm{L}$ to $278.36 \mu \mathrm{mol} / \mathrm{L}$, indicating that the ferric reducing power of PSPW was significantly enhanced.

(A)

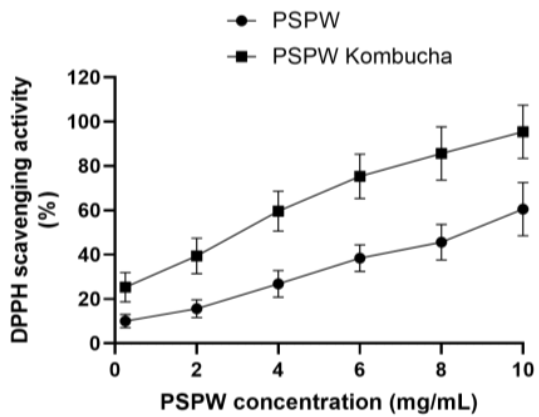

(B)

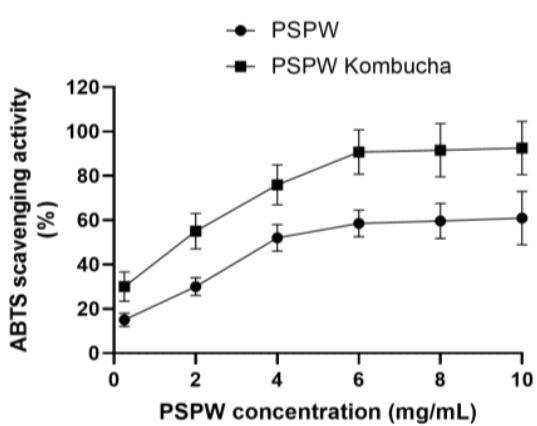

(C)

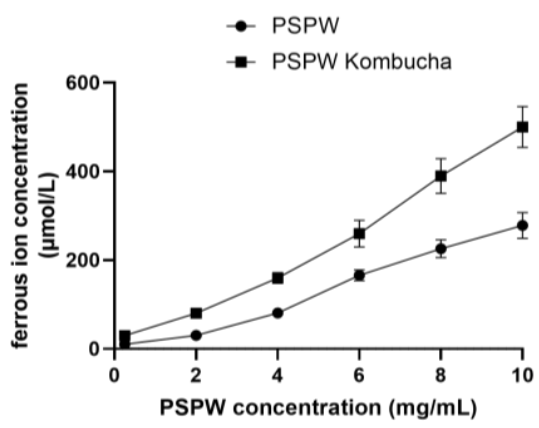

Fig. 5 In vitro antioxidant activity of $80 \%$ methanol extracts from unfermented and fermented PSPW. Data were shown as the mean $\pm \operatorname{SD}(n=3)$.

\section{Conclusion}

In this study, a new type of kombucha beverage with functional activity was obtained by using kombucha as strain and PSPW as substrate for fermentation. The results of this study can support the development of a kombucha fermented beverage with improved nutritional value and functional characteristics. The nutritional and functional properties of PSPW after fermentation were significantly improved compared with those before fermentation.

\section{Acknowledgments}

This project was funded by Grants from the Guiding Project in Science Research Program from Education Department of Hubei (B2020222).

\section{References}

1. Tran Thierry., Grandvalet Cosette., Verdier François., Martin Antoine., Alexandre Hervé., Tourdot-Maréchal Raphaëlle.(2020). Microbiological and technological parameters impacting the chemical composition and sensory quality of kombucha. Compr Rev Food Sci Food Saf, 19(4), 2050-2070.

2. Mousavi Seyyed Mojtaba,Hashemi Seyyed Alireza,Zarei Maryam et al. Recent Progress in Chemical Composition, Production, and Pharmaceutical Effects of Kombucha Beverage: A Complementary and Alternative Medicine.[J] .Evid Based Complement Alternat Med, 2020, 2020: 4397543.

3. Wang Shengguang,Zhang Shiming, Wang Shaoping et al. A comprehensive review on Pueraria: Insights on its chemistry and medicinal value.[J] .Biomed Pharmacother, 2020, 131: 110734.

4. Sengupta S,Jana M L,Sengupta D et al. A note on the estimation of microbial glycosidase activities by dinitrosalicylic acid reagent.[J] .Appl Microbiol Biotechnol, 2000, 53: 732-5.

5. Chakravorty Somnath,Bhattacharya Semantee, Chatzinotas Antonis et al. Kombucha tea fermentation: Microbial and biochemical dynamics.[J] .Int J Food Microbiol, 2016, 220: 6372. 